\title{
Pares articulares en prótesis primaria de cadera. ¿Existe alguna diferencia?
}

\author{
Bearing surfaces in primary hip arthroplasty. Is there any difference? \\ Suárez JC, ${ }^{*}$ Forero A,* Llinás A, ${ }^{*, \star}$ Bonilla $G,{ }^{*, \star}$ Rodríguez $H,{ }^{\S}$ Amado O" \\ Hospital Universitario Fundación Santa Fe.
}

RESUMEN. Introducción: En un reemplazo total de cadera primario (RTC), diferentes materiales son los que se usan para las superficies de apoyo. En nuestro medio, las cabezas de metal o cerámica con polietileno altamente entrecruzado (PA) son los más utilizados. Estas combinaciones tienen buenos resultados, pero no es claro cuál es superior clínicamente. Basados en una revisión sistemática de la literatura y de los registros nacionales de artroplastía, el objetivo de este estudio es determinar si existe alguna diferencia significativa desde el punto de vista clínico. Material y métodos: Realizamos una revisión sistemática de la literatura y de los registros nacionales de artroplastía. Buscamos estudios en los que se compararan los tipos de superficie de contacto: cerámica-polietileno altamente entrecruzado (CP) y metal-polietileno altamente entrecruzado (MP), además de los registros nacionales de artroplastía que describieran, con un seguimiento mínimo de 10 años, la tasa de revisión según el tipo de superficie. El desenlace evaluado fue: tasa de revisión por cualquier causa según el tipo de superficie. Resultados: Dos de quince registros nacionales fueron incluidos: el registro australiano muestra una diferencia en la tasa de revisión a 15 años, comparando CP: 6.3 (IC 5.8, 6.7) contra MP: 5.1 (IC 4.6, 5.7). El registro de Nueva Zelanda no muestra diferencias en la tasa de revisión/100 componentes/ año: CP de 0.54 (0.48-0.61) en comparación con MP de
ABSTRACT. Introduction: In primary hip replacement, different materials are used for bearing surfaces. In our medium metal or ceramic heads with highly crossed-linked polyethylene (PA) are the most used. These combinations have good results, but it is not clear which is clinically superior. The objective of this study is to determine whether there is any clinically significant difference based on a systematic review of the literature and national registries of arthroplasty. Material and methods: We conduct a systematic review of the literature and national registries of arthroplasty and we were looking for studies comparing bearing surfaces: ceramic-highly cross-linked polyethylene (CP) and metal-highly cross-linked polyethylene (MP); describing the revision rate according to the surface type with a minimum 10-year follow-up. The outcome evaluated was: review rate for any cause depending on surface type. Results: Two out of fifteen national registries were included. The Australian registry shows a difference in the 15-year revision rate: CP: 6.3 (IC 5.8, 6.7) vs MP: 5.1 (IC 4.6, 5.7). The New Zealand registry shows no differences in revision rate/100 components/year: CP 0.54 (0.48-0.61) vs MP 0.61 (0.57-0.66). We do not find clinical studies with inclusion criteria that answer the research question. Conclusion: The results of this review show a high survival rate with the use of highly cross-linked polyethylene, the results are similar when using ceramic or metal heads.

\section{Nivel de evidencia: III}

* Departamento de Ortopedia y Traumatología, Hospital Universitario de la Fundación Santa Fe de Bogotá. Bogotá, D.C., Colombia.

${ }^{\ddagger}$ Facultad de Medicina, Universidad de Los Andes. Facultad de Medicina, Universidad del Rosario. Bogotá, D.C., Colombia.

$\S$ Departamento de Ortopedia y Traumatología, Hospital Infantil de San José. Facultad de Medicina, Fundación Universitaria de Ciencias de la Salud. Bogotá, D.C., Colombia.

" Departamento de Ortopedia y Traumatología, Fundación Oftalmológica FOSCAL. Bucaramanga, Colombia.

Dirección para correspondencia:

Dra. Cristina Suárez J.

Departamento de Ortopedia y Traumatología, Hospital Universitario de la Fundación Santa Fe de Bogotá.

Carrera 7 Núm. 117-15, C.P. 110111186, Bogotá, D.C., Colombia.

Teléfono: 6030303, ext. 5832

E-mail: cristinasuarezj@gmail.com 
0.61 (0.57-0.66). No encontramos estudios clínicos con los criterios de inclusión que respondan la pregunta de investigación. Conclusión: Los resultados de esta revisión muestran una alta supervivencia cuando se usa polietileno altamente entrecruzado; asimismo, los resultados son similares cuando se utilizan cabezas de cerámica o metálicas.

Palabras clave: Reemplazo, cadera, supervivencia, cerámica, metal, polietileno.

\section{Introducción}

El reemplazo total de cadera (RTC) se ha establecido como el procedimiento más efectivo para corregir la artrosis degenerativa de la cadera y ha demostrado mejorar de forma satisfactoria la calidad de vida, manteniendo la costo-efectividad tanto para el paciente como para la sociedad. ${ }^{1,2}$ Con el ánimo de mejorar la función y la supervivencia de los implantes, éste ha sufrido varios cambios en sus diseños y materiales. Uno de los componentes del RTC que persiste en la actualidad en debate es la superficie de apoyo. En consecuencia, el desarrollo del polietileno altamente entrecruzado (PA) sigue demostrando su duración a largo plazo con un mínimo desgaste y osteólisis. ${ }^{3}$ En Estados Unidos y muchos otros países, los insertos acetabulares más usados son de PA, pues su superioridad comparado con el polietileno convencional está respaldada por estudios con simuladores, que reportan menor penetración femoral y desgaste en el PA. ${ }^{4,5}$ Otros estudios clínicos aleatorizados donde se evalúa radiológicamente el desgaste de los insertos, de igual manera, muestran menor desgaste en PA. También algunos metaanálisis y revisiones sistemáticas sugieren una menor penetración y desgaste, así como estudios basados en los registros nacionales, los cuales demuestran menores tasas de revisión en RTC con insertos de PA. ${ }^{6,7,89}$ Sin embargo, la pregunta sobre cuál es el mejor material de las cabezas femorales con el que se pueda combinar el polietileno altamente entrecruzado sigue sin responderse.

A nivel mundial, en los RTC, las superficies de apoyo más usadas son las cabezas de metal o cerámica articuladas con PA. ${ }^{7,10}$ En los últimos años, el uso de cabezas de cerámica en RTC ha venido en aumento, debido a los buenos resultados de las características de desgaste cuando se combinan con cerámica o PA y a que las cerámicas modernas permiten el uso de cabezas de grandes diámetros sin que se aumente la fricción o el desgaste y sin mayores problemas en la unión con los conos. ${ }^{8}$

Poco se conoce sobre la efectividad al contrastar las cabezas de cerámica con las metálicas sobre los insertos de
Keywords: Replacement, hip, survival, ceramic, metal, polyethylene.

PA; sólo un registro nacional compara estas dos superficies con el PA. ${ }^{8}$ La cerámica tiene ventajas teóricas in vitro al compararlas con las cabezas de metal, ya que su terminado liso, su dureza y capacidad de lubricación conllevan a una menor fricción, mayor lubricación, menor posibilidad de roce y, por consiguiente, menores tasas de desgaste y osteólisis en los insertos. ${ }^{11}$

Por tanto, el propósito de este estudio es responder a la siguiente pregunta: ¿existen diferencias en las tasas de supervivencia a largo plazo entre las combinaciones de cerámica-polietileno altamente entrecruzado y metal-polietileno altamente entrecruzado?

\section{Material y métodos}

Se realizó una revisión sistemática de la literatura y los registros de artroplastías para identificar aquellos estudios que verificaran las combinaciones de interés o los registros que reportaran datos comparativos. Como criterios de inclusión, para el análisis se tuvieron en cuenta aquellos estudios clínicos que compararan la supervivencia de las dos combinaciones de interés con un seguimiento mínimo de 10 años. En cuanto a los registros, se decidió incluir el último reporte de aquellos registros nacionales de artroplastía en los que se describiera la tasa de revisión de RTC a un mínimo de 10 años, se incluyeran todos los tipos de fijación (cementada, no cementada, híbrida, híbrida reversa) y se especificara el tipo de polietileno en el reemplazo.

Tres de los autores (OA, AF, CS) realizaron la búsqueda de los estudios clínicos en pacientes mayores de 18 años que compararan la supervivencia o las tasas de revisión de RTC con las combinaciones cerámica-PA y metal-PA con un seguimiento mínimo de 10 años.

Para ello, se revisaron las siguientes bases de datos: PubMed, Ovid MEDLINE, Ovid EMBASE y Clinical Trials, con las siguientes palabras clave y términos MeSH: «hip arthroplasty», «hip replacement», «ceramic alumina», «ceramic», «metal alloys», «metal», «chromium», «polyethylene, «highly cross-linked».

La supervivencia del implante o la tasa de revisión por cualquier causa en el último seguimiento fue el único re- 
sultado evaluado. De este modo, se revisó el último reporte de 15 registros nacionales de artroplastías.

\section{Resultados}

Dentro de la búsqueda sistemática de estudios clínicos, no se encontró ningún resultado que comparase las superficies de apoyo metal-PA y cerámica-PA. Tan sólo dos de los registros nacionales: el registro australiano y el registro de Nueva Zelanda cumplieron los criterios de inclusión.

El registro australiano y el registro de Nueva Zelanda son los únicos registros nacionales de artroplastía en los que se reporta específicamente la tasa de revisión con un seguimiento de más de 10 años de RTC con cerámica-PA y metal-PA. ${ }^{8,12}$ En el seguimiento a 10 años del registro australiano, no se encontraba ninguna diferencia estadísticamente significativa al comparar estas dos superficies de apoyo cerámica-PA: 4.5 (IC 4.1, 4.8) frente a metalPA: 4.4 (IC 4.3, 4.6); pero a los 15 años de seguimiento, apareció una diferencia en la tasa de revisión cerámicaPA de 5.1 (IC 4.6, 5.7) frente a metal-PA de 6.3 (IC 5.8, 6.7) (Tabla 1). ${ }^{8}$

En el último registro de Nueva Zelanda, la tasa/100 componentes/año en la combinación cerámica-PA fue de 0.54 (0.48-0.61) comparada con 0.61 (0.57-0.66) en la combinación metal-PA, en la cual tampoco se encontró una diferencia estadísticamente significativa (Tabla 2). ${ }^{12}$

En el resto de los registros revisados, no hubo información sobre el tipo de superficie. Sin embargo, en el registro británico, la información se encuentra relacionada por grupos etarios y tipos de cabeza femoral, pero no se diferencian por el tipo de polietileno (convencional o polietileno altamente entrecruzado), por lo que no fue incluido en el análisis.

\section{Discusión}

Dado el número creciente de RTC y de pacientes cada vez más jóvenes que requieren este procedimiento, aparece la necesidad de mejorar la durabilidad de los implantes para este procedimiento. En ese esfuerzo, se cree que las cabezas de cerámica con insertos de polietileno altamente entrecruzado pueden mostrar una mejoría en las tasas de revisión a largo plazo.

En los estudios de laboratorio, las cabezas de cerámica muestran una mejor lubricación y son más resistentes al desgaste cuando se comparan con las cabezas de metal; ${ }^{13,14,15}$ sin embargo, estas ventajas aún no son claras en el contexto clínico.

En este trabajo, pudimos identificar dos registros que permiten comparar los resultados a largo plazo. Uno de ellos no mostró diferencias a 10 años y el otro mostró una diferencia de $1.2 \%$ a 15 años, lo que favorecería el uso de las cabezas de cerámica. Para nuestro conocimiento, ésta es la primera vez que una revisión de este tipo identifica diferencias entre estos dos pares de fricción.

La primera observación de este trabajo es que los insertos de polietileno altamente entrecruzado se asocian con bajas tasas de falla ( $<10 \%$ a 15 años), hallazgo

\begin{tabular}{|c|c|c|c|c|c|c|c|c|}
\hline Superficie de apoyo & Revisado (n) & Total (n) & 1 año & 3 años & 5 años & 10 años & 15 años & 16 años \\
\hline Cerámica/cerámica & 2,758 & 78,674 & $1.5(1.4-1.6)$ & $2.4(2.3-2.5)$ & $3.1(3.0-3.2)$ & $5.0(4.8-5.2)$ & $7.2(6.8-7.7)$ & $7.3(6.9-7.8)$ \\
\hline $\begin{array}{l}\text { Cerámica/polietileno no } \\
\text { altamente entrecruzado }\end{array}$ & 429 & 6,288 & $1.8(1.5-2.2)$ & $3.0(2.6-3.5)$ & $3.7(3.3-4.3)$ & $7.0(6.2-7.8)$ & $11.9(10.7-13.2)$ & $13.1(11.6-14.7)$ \\
\hline $\begin{array}{l}\text { Cerámica/polietileno } \\
\text { altamente entrecruzado }\end{array}$ & 1,276 & 49,627 & $1.6(1.5-1.8)$ & $2.5(2.3-2.7)$ & $3.1(2.9-3.3)$ & $4.5(4.1-4.8)$ & $5.1(4.6-5.7)$ & \\
\hline Cerámica/metal & 18 & 299 & $1.7(0.7-4.0)$ & $3.7(2.1-6.6)$ & $4.4(2.6-7.4)$ & & & \\
\hline Metal/metal & 347 & 5,146 & $1.6(1.3-1.9)$ & $3.3(2.9-3.9)$ & $4.4(3.8-5.0)$ & $6.5(5.8-7.2)$ & $8.7(7.7-9.7)$ & $9.1(8.0-10.4)$ \\
\hline $\begin{array}{l}\text { Metal/polietileno no } \\
\text { altamente entrecruzado }\end{array}$ & 2,310 & 34,593 & $1.4(1.3-1.5)$ & $2.4(2.3-2.6)$ & $3.4(3.2-3.6)$ & $6.3(6.1-6.6)$. & $10.5(10.1-11.0)$ & $11.3(10.7-11.9)$ \\
\hline $\begin{array}{l}\text { Metal/polietileno altamente } \\
\text { entrecruzado }\end{array}$ & 3,999 & 131,327 & $1.6(1.5-1.6)$ & $2.3(2.2-2.4)$ & $2.9(2.8-3.1)$ & $4.4(4.3-4.6)$ & $6.3(5.6-6.7)$ & $6.3(5.8-6.7)$ \\
\hline $\begin{array}{l}\text { Metal ceramizado/ } \\
\text { polietileno no altamente } \\
\text { entrecruzado }\end{array}$ & 36 & 290 & $1.7(0.7-4.1)$ & $3.9(2.2-6.9)$ & $4.3(2.4-7.4)$ & $12.5(8.9-17.5)$ & & \\
\hline $\begin{array}{l}\text { Metal ceramizado/ } \\
\text { polietileno altamente } \\
\text { entrecruzado }\end{array}$ & 426 & 18,177 & $1.6(1.4-1.7)$ & $2.0(1.8-2.3)$ & $2.3(2.1-2.6)$ & $3.4(3.0-3.8)$ & & \\
\hline Total & 11,599 & 324,421 & & & & & & \\
\hline
\end{tabular}


Tabla 2: Comparación revisiones polietileno estándar en comparación con polietileno altamente entrecruzado.

\begin{tabular}{|c|c|c|c|c|c|}
\hline Superficies & Procedimientos (n) & $\begin{array}{c}\text { Componentes } \\
\text { observados por año }\end{array}$ & Revisado (n) & Tasa/100 componentes año & Intervalo de confianza (95\%) \\
\hline $\mathrm{CC}$ & 11,235 & $58,591.1$ & 355 & 0.61 & $0.54-0.67$ \\
\hline $\mathrm{CM}$ & 474 & $2,601.6$ & 21 & 0.81 & $0.50-1.23$ \\
\hline $\mathrm{CP}$ & 21,242 & $118,266.3$ & 769 & 0.65 & $0.61-0.70$ \\
\hline PS & 6,833 & $65,690.0$ & 486 & 0.74 & $0.68-0.81$ \\
\hline PX & 14,382 & $52,521.9$ & 283 & 0.54 & $0.48-0.61$ \\
\hline MM & 5,989 & $55,702.6$ & 797 & 1.43 & $1.33-1.53$ \\
\hline MP & 67,226 & $437,443.2$ & 3,018 & 0.69 & $0.67-0.71$ \\
\hline PS & 35,647 & $295,137.1$ & 2,143 & 0.73 & $0.70-0.76$ \\
\hline PX & 31,579 & $142,306.0$ & 875 & 0.61 & $0.57-0.66$ \\
\hline
\end{tabular}

que es independiente del material de la cabeza femoral utilizada. La segunda conclusión es que las cabezas de cerámica y metal producen una mínima o ninguna diferencia en las tasas de supervivencia al ser utilizadas en la población general. Esta conclusión es consistente con lo reportado recientemente por Cafri y otros en su estudio retrospectivo del registro Kaiser Permanente National Total Joint Replacement Registry (TJRR) de Estados Unidos en 2017, donde se compararon artroplastías no cementadas con cabezas de metal o de cerámica de diferentes tamaños (<32, 32, $36>36 \mathrm{~mm})$. Este estudio no muestra diferencias significativas en la tasa de revisión por cualquier causa. ${ }^{16}$

La principal fortaleza de este estudio es el alto número de pacientes incluidos en los registros, lo cual aumenta el poder estadístico para encontrar diferencias de poca magnitud. Las principales limitaciones son, en primer lugar, la falta de estudios clínicos comparativos que permitan contrarrestar los sesgos de selección propios de los registros y, en segundo lugar, la imposibilidad de realizar análisis por grupos de edad en los registros incluidos. Esta última dificultad es en especial importante, dado que las fallas relacionadas con el desgaste podrían ser más frecuentes en pacientes jóvenes, lo cual produciría una diferencia entre los resultados de los dos pares de fricción en este grupo.

En conclusión, basados en estos resultados, aún no podemos hacer una recomendación sobre cuál de las cabezas, si de cerámica o de metal, es la mejor para la población general. Su utilización debe estar definida por las preferencias del cirujano y expectativas del paciente.

Bibliografía

1. Koenig L, Zhang Q, Austin MS, Demiralp B, Fehring TK, Feng C, et al. Estimating the societal benefits of THA after accounting for work status and productivity: a Markov model approach. Clin Orthop Relat Res. 2016; 474(12): 2645-54.

2. Keurentjes JC, Van Tol FR, Fiocco M, Schoones JW, Nelissen RG. Minimal clinically important differences in health-related quality of life after total hip or knee replacement: a systematic review. Bone Joint Res [Internet]. 2012; 1(5): 71-7. Disponible en: http://www. pubmedcentral.nih.gov/articlerender.fcgi?artid=3626243\&tool=pmce ntrez\&rendertype $=$ abstract

3. Babovic N, Trousdale RT. Total hip arthroplasty using highly cross-linked polyethylene in patients younger than 50 years with minimum 10-year follow-up. J Arthroplasty [Internet]. 2013; 28(5): 815-7. Disponible en: http://linkinghub.elsevier.com/retrieve/pii/ S0883540313001113

4. Geerdink CH, Grimm B, Ramakrishnan R, Rondhuis J, Verburg AJ, Tonino AJ. Crosslinked polyethylene compared to conventional polyethylene in total hip replacement: pre-clinical evaluation, in vitro testing and prospective clinical follow-up study. Acta Orthop. 2006; 77(5): 719-25.

5. Muratoglu OK, Bragdon CR, O’Connor DO, Jasty M, Harris WH. A novel method of cross-linking ultra-high-molecular-weight polyethylene to improve wear, reduce oxidation, and retain mechanical properties. J Arthroplasty [Internet]. 2001; 16(2): 149-60. Disponible en: http://linkinghub.elsevier.com/retrieve/pii/S0883540301361466

6. Digas G, Kärrholm J, Thanner J, Malchau H, Herberts P. The Otto Aufranc Award. Highly cross-linked polyethylene in total hip arthroplasty: randomized evaluation of penetration rate in cemented and uncemented sockets using radiostereometric analysis. Clin Orthop Relat Res. 2004; (429): 6-16.

7. Bozic KJ, Kurtz S, Lau E, Ong K, Chiu V, Vail TP, et al. The epidemiology of bearing surface usage in total hip arthroplasty in the United States. J Bone Joint Surg Am [Internet]. 2009; 91(7): 1614-20. Available from: http://content.wkhealth.com/linkback/openurl?sid=W KPTLP:landingpage\&an=00004623-200907000-00008

8. Green M, Wishart N, Young E, Mccormack V, Swanson M. 14th Annual Report. National Joint Registry for England, Wales, Northern Ireland and the Isle of Man. The NJR Editorial Board. 2017 [December 2016].

9. Manning DW, Chiang PP, Martell JM, Galante JO, Harris WH. In vivo comparative wear study of traditional and highly cross-linked polyethylene in total hip arthroplasty. J Arthroplasty. 2005; 20(7): 880-6.

10. McKesson Corporation [Internet]. Annual Report 2017. New York: McKesson Corporation. 2017. Disponible en: http://www. annualreports.com/HostedData/AnnualReportArchive/m/NYSE_ MCK_2017.pdf

11. Semlitsch M, Lehmann M, Weber H, Doerre E, Willert HG. New prospects for a prolonged functional life-span of artificial hip joints by using the material combination polyethylene/aluminum oxide ceramic/metal. J Biomed Mater Res. 1977; 11(4): 537-52.

12. New Zealand Orthopaedic Association. The New Zealand Joint Registry Seventeen Year Report (January 1999-December 2015) [Internet]. Editorial Committee. 2016. Disponible en: www.nzoa.org. nz/nz-joint-registry 
13. Meftah M, Klingenstein GG, Yun RJ, Ranawat AS, Ranawat CS. Longterm performance of ceramic and metal femoral heads on conventional polyethylene in young and active patients. J Bone Joint Surg Am [Internet]. 2013; 95(13): 1193-7. Disponible en: http://content.wkhealth.com/linkback/ openurl?sid=WKPTLP:landingpage\&an=00004623-201307030-00006

14. Clarke IC, Gustafson A. Clinical and hip simulator comparisons of ceramic-on-polyethylene and metal-on-polyethylene wear. Clin Orthop Relat Res. 2000; (379): 34-40.
15. Boutin P. Total arthroplasty of the hip by fritted alumina prosthesis. Experimental study and 1st clinical applications. Orthop Traumatol Surg Res [Internet]. 2014; 100(1): 15-21. Available from: http://dx.doi. org/10.1016/j.otsr.2013.12.004

16. Cafri G, Ma EWP, Mph RL, Bini SA, Kurtz SM. Is there a difference in revision risk between metal and ceramic heads on highly crosslinked polyethylene liners? Clin Orthop Relat Res. 2017; 475(5): 1349-55. 